\title{
SOBRE LA EVOLUCIÓN COMO CIENCIA DE LA GEOMORFOLOGÍA DINÁMICA EN AMBIENTES FLUVIALES
}

\author{
Jesús Horacio \\ Departamento de Geografía. Universidad de Concepción. Chile \\ Instituto de Investigacións Tecnolóxicas. Universidade de Santiago de Compostela \\ horacio.garcia@usc.es
}

\section{RESUMEN}

Se presenta un análisis histórico-evolutivo de la geomorfología dinámica en ambientes fluviales. El objetivo del estudio se centra en organizar los hechos temporales pasados que han ido confeccionando el cuerpo de lo que actualmente es esta ciencia. La línea del tiempo establecida se estructura en diferentes etapas, cada una de las cuales se define por unas características propias que derivan en una forma concreta de practicar y concebir la ciencia. Dicha línea del tiempo actúa también como guía y argumento en la lectura del texto.

Palabras clave: geomorfología fluvial, antecedentes, historia, ciencia, evolución.

\section{ABSTRACT}

This paper analyses the history and development of fluvial geomorphology in dynamic environments. The goal of the study is to organize the past events that have been making up the body of what this science is now. The established timeline is divided into different stages, each of which is defined by its own characteristics resulting in a particular way to conceive of and practice science; the timeline also serves to guide the reading of this text.

Keywords: fluvial geomorphology, background, history, science, development.

Fecha de recepción: mayo 2015.

Fecha de aceptación: marzo 2016. 


\section{INTRODUCCIÓN}

El desarrollo del presente trabajo tiene el objetivo de organizar los devenires históricos de la geomorfología dinámica en ambientes fluviales (Horacio, 2014). Con ello se obtiene una mejor contextualización y comprensión de lo que actualmente es esta ciencia. Algunos estudios aluden a las ventajas de tener un conocimiento global de la ciencia que se practica (Sack, 2002), lo que también otorga al investigador/a un mayor criterio pragmático. A fin de cuentas, el contexto temporal actual está determinado por hechos evolutivos pasados.

Sobre este propósito general del estudio subyacen tres aspectos que es preciso aclarar. Primero, que la geomorfología fluvial es la ciencia encargada de estudiar las formas y procesos de los ríos (ver Tabla 1), pudiéndolo hacer sobre el momento actual (presente) o en su contexto histórico (pasado). La línea de trabajo de este estudio está relacionada solo con la parte de la geomorfología fluvial centrada en la dinámica actual. Segundo, que los antecedentes, que se exponen de forma evolutiva, son polivalentes en sus inicios. Tercero, que el uso del término geomorfología fluvial en este texto hace referencia a los procesos y formas actuales, por consiguiente, sin la perspectiva cuaternarista o histórica. Con todo, es preciso indicar que actualmente existe una notable cantidad de trabajos que tratan de geomorfología fluvial pleistocena y holocena. Y que además han ido en aumento a medida que los métodos de datación van permitiendo situar cambios en la dinámica fluvial, marcados por fases de agradación y degradación y su relación con cambios climáticos, tectónica, etc. (Stokes et al., 2012; Geach et al., 2014).

El término geomorfología surgió en el Servicio Geológico de los EE.UU. en la década de 1880 y fue posiblemente acuñado por dos grandes pioneros en el campo, J.W. Powell y W.J. McGee (Hart, 1986). En Europa, el uso generalizado de la palabra morfología aplicada a las formas de la superficie de la Tierra se debe a la obra de A. Penck (1894) Morfologie der Erdoberfläche (George, 2007). Según su epistemología la geomorfología proviene del griego y está compuesta por dos términos: geo (del lat. geos (Tierra), y este del gr. $\mathrm{cu}$ ) + morfología, a su vez formada por dos nuevos conceptos, morfo (del gr.

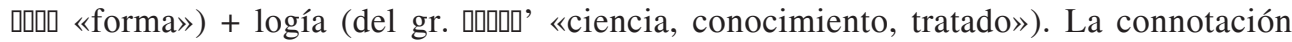
"fluvial" engloba todo aquello perteneciente o relativo al río. De este modo, la geomorfología se trata de una rama de la geografía física y/o geología dedicada al estudio de los geoprocesos y geoformas presentes en la superficie de la Tierra. En tanto la geomorfología fluvial (Tabla 1) se centrará en el conocimiento de las formas (morfología) y procesos (dinámica) derivados de los ríos.

Tabla 1

DIVERSAS DEFINICIONES DE GEOMORFOLOGÍA FLUVIAL

\begin{tabular}{|l|l|}
\hline \multicolumn{1}{|c|}{ Definición } & \multicolumn{1}{|c|}{ Fuente } \\
\hline $\begin{array}{l}\text { La geomorfología fluvial tiene como objeto de estudio el sistema de drenaje en su } \\
\text { conjunto así como los canales que individualmente lo conforman. }\end{array}$ & $\begin{array}{l}\text { Kruska y } \\
\text { Lamarra (1973) }\end{array}$ \\
\hline $\begin{array}{l}\text { Estudio de los procesos de erosión y deposición generados por la escorrentía } \\
\text { superficial formados a partir del agua precipitada sobre un territorio. }\end{array}$ & Schumm (1977) \\
\hline
\end{tabular}




\begin{tabular}{|c|c|}
\hline $\begin{array}{l}\text { El objetivo principal de la geomorfología fluvial se ha de centrar en la explicación } \\
\text { de las relaciones entre los procesos físicos del flujo en canales de lecho móvil, la } \\
\text { mecánica del transporte de sedimentos (desplazados por el flujo) y las formas de } \\
\text { los canales aluviales creados por el transporte de sedimentos. }\end{array}$ & Richards (1987) \\
\hline $\begin{array}{l}\text { La geomorfología fluvial es el estudio de las formas y procesos de la superficie } \\
\text { terrestre derivados de fenómenos fluviales y/o relacionados con la escorrentía } \\
\text { superficial. }\end{array}$ & Graf (1988) \\
\hline $\begin{array}{l}\text { La geomorfología fluvial se centra en el estudio de los cambios ocurridos en el } \\
\text { cauce. Se trata de una ciencia "de campo", clasificación y descripción. }\end{array}$ & Petts (1995) \\
\hline $\begin{array}{l}\text { La geomorfología fluvial se centra en el estudio de las formas y procesos de los } \\
\text { ríos. Más concretamente en el trabajo de erosión y transporte de caudal líquido y } \\
\text { sólido que los ríos realizan a través de la red de drenaje. }\end{array}$ & $\begin{array}{l}\text { Knighton } \\
\text { (1998) }\end{array}$ \\
\hline $\begin{array}{l}\text { Ciencia que trata de investigar la complejidad del comportamiento de los cauces } \\
\text { fluviales en un rango de escala a nivel de sección y de cuenca, pero también busca } \\
\text { investigar el proceso-respuesta a una escala de tiempo larga, aunque por lo general } \\
\text { dentro de unos ciclos climáticos recientes. }\end{array}$ & $\begin{array}{l}\text { Newson y Sear } \\
\text { (1998) }\end{array}$ \\
\hline $\begin{array}{l}\text { La geomorfología fluvial es una disciplina multidisciplinar encargada del estudio } \\
\text { delos ríos como sistemas complejos. }\end{array}$ & Dollar (2000) \\
\hline $\begin{array}{l}\text { La geomorfología fluvial es una disciplina de síntesis con raíces en geología, } \\
\text { geografía e ingeniería de ríos, las cuales se apoyan en campos tales como la } \\
\text { hidrología, química, física, ecología, ciencias sociales e historia natural. }\end{array}$ & $\begin{array}{l}\text { Kondolf y } \\
\text { Piégay (2003) }\end{array}$ \\
\hline $\begin{array}{l}\text { La geomorfología fluvial es el área de estudio dedicada a la comprensión de las } \\
\text { formas terrestres originadas por los ríos. }\end{array}$ & Bouier (2004) \\
\hline $\begin{array}{l}\text { La geomorfología fluvial es, estrictamente, la geomorfología de los ríos, es decir, } \\
\text { el área de estudio de las formas del terreno producidas por estos. }\end{array}$ & Gregory (2004) \\
\hline $\begin{array}{l}\text { Ciencia que proporciona el marco físico sobre el que subyace el paisaje que } \\
\text { sustenta la integridad ecológica de los sistemas fluviales. }\end{array}$ & $\begin{array}{l}\text { Brierley y } \\
\text { Fryirs (2005) }\end{array}$ \\
\hline $\begin{array}{l}\text { La geomorfología fluvial es el estudio de las interacciones entre las formas y } \\
\text { procesos del río dentro de una escala de espacio y tiempo. }\end{array}$ & Charlton (2008) \\
\hline $\begin{array}{l}\text { La geomorfología fluvial estudia la historia de las formas del río, tratando de } \\
\text { entender sus procesos y predecir su dinámica, combinando para ello observaciones } \\
\text { de campo, estudios experimentales y modelos numéricos. }\end{array}$ & $\begin{array}{l}\text { Thorndycraft et } \\
\text { al. (2008) }\end{array}$ \\
\hline
\end{tabular}

La geomorfología, y más concretamente su variante fluvial, es una ciencia incipiente que experimentó un fuerte impulso en los últimos 60 años (Gregory, 2004). Para algunos autores el nacimiento de la geomorfología se produce con la publicación de la obra The Geographical Cycle (Davis, 1899), aunque fuera de esta fecha concreta, el auténtico nacimiento de la geomorfología como ciencia se da cuando abandona la descripción y comienza a analizar y explicar (Gutiérrez, 2008). Esta concepción científica de la geomorfología, en el sentido que hoy se entiende, no comienza a atisbarse hasta el siglo XVIII, muy especialmente en las últimas décadas (Fig. 1). Previa a esta fecha la evolución de la geomorfología, como sucede en la mayor parte de las ciencias, está íntimamente ligada a los devenires filosóficos y religiosos de cada período histórico (Glacken, 1996). 


\section{METODOLOGÍA}

El proceso metodológico seguido está basado en un amplio vaciado bibliográfico sobre el tema en cuestión. Finalizada esta etapa inicial se ha procedido con la lectura pormenorizada de las principales obras. Con la información extraída se ha establecido una línea del tiempo que conduce al lector hasta los orígenes de la geomorfología fluvial como ciencia, desde la concepción inicial, pasando por sus muy diversas etapas hasta concluir en la geomorfología fluvial del siglo XXI. Estructuralmente se ha seguido la historia de la geomorfología en general, aunque enfatizando en aquello de mayor relevancia para el ámbito de la morfodinámica fluvial (autores, publicaciones, hitos, momentos, etc.).

El catálogo de fuentes consultada ha sido amplio. De entre estas destacan las siguientes obras: Tricart (1965), Schumm (1972), Pitty (1982), Sala (1982, 1984), Capel (1983), Grau y Sala (1984), Beckinsale y Chorley (1991), Strahler (1992), Tinkler (1985), Glacken (1996), Pedraza (1996), Sala y Batalla (1996), Sack (2002), Bauer (2004), Gutiérrez (2008) o Oldroyd y Grapes (2008), entre otras. Todos estos trabajos describen sintética, pero profusamente, parte de la historia de la geomorfología. Existen muchas otras obras no citadas que también abordan la temática de la geomorfología en su contexto histórico. Las referencias nominadas son unas dentro de muchas otras, pero se consideró que estas ofrecían una información lo suficientemente relevante como para confeccionar el actual trabajo.

Es también necesario matizar que el límite temporal asignado a cada etapa histórica hay que verlo en un sentido flexible. Por este motivo se citan obras que pueden rebasar, de un modo leve, los límites temporales establecidos. Así, por ejemplo, en el periodo que va desde finales del siglo XIX hasta finales del siglo XX se incluyen obras de la década del 2000. El paso de un periodo a otro es un proceso gradual en el que durante un tiempo conviven las tendencias de cada etapa.

\section{DESARROLLO EVOLUTIVO DE LA GEOMORFOLOGÍA FLUVIAL COMO CIENCIA}

\section{III.1. La Geomorfología Fluvial previa al siglo XX}

Es posible diferenciar dos etapas en la evolución histórica de la ciencia geomorfológica hasta el siglo XVIII: (1) fase correspondiente con la antigüedad clásica greco-latina (siglos V-VI a.C. hasta la Era Cristiana), donde se mezclan las primeras aportaciones de naturalistas y cartógrafos que tratan de buscar un porqué racional a las observaciones naturales, pero con ideologías más fantasiosas y frecuente recurrencia a los dioses para dar respuesta a la creación natural; (2) fase comprendida entre, aproximadamente, el Anno Domini y el Renacimiento (s. XV-XVI), aunque el fuerte fanatismo religioso dominante en estos siglos, bajo el principio absoluto de Creación Divina, se posterga en el tiempo hasta los albores del siglo XIX. En el final de este periodo gobiernan las hipótesis catastrofistas como explicativas de los cambios geológicos acaecidos en el Planeta. Entre los siglos XVII-XVIII-XIX se establece un periodo de transición hacia la geomorfología moderna (ver Fig. 1). Durante estos siglos las ideas diluvianas comienzan a perder fuerza ante el empuje de trabajos con base científica de la mano de pioneros estudiosos como: Leonardo da Vinci (1452-1519), Palissy 
(1510-1590), Perrault (1608-1680), Mariotte (1620-1684), Halley (1656-1742), Pitot (16951771), Bernoulli (1654-1705), Euler (1707-1783), Guettard (1715-1786), de Chézy (17181798), Desmarest (1725-1815), De Saussure (1740-1799), Lamarck (1744-1829), Manning (1816-1897) o Surell (1813-1887), por citar algunos, que tratan de analizar y comprender formas y procesos diversos. Será la obra de James Hutton (1795), Theory of the Earth, la que de paso al principio del uniformismo, desbancando de este modo el paradigma catastrofista enunciado por Cuvier (1769-1832), quien defendía que los cambios geológicos ocurridos en el Planeta se debían a bruscos y repentinos episodios. Frente a ello, la teoría del uniformismo postulaba que los procesos naturales que actuaron en el pasado son los mismos que actúan en el presente. Cabe destacar también el trabajo de Playfair, Illustrations of the Huttonian Theory of the Earth (1802), que sirvió para divulgar y consolidar las ideas previamente descritas por Hutton sobre la permanencia de las causas actuales de la Tierra durante su evolución.

Figura 1

ETAPAS HISTÓRICAS EN LA EVOLUCIÓN DE LA GEOMORFOLOGÍA

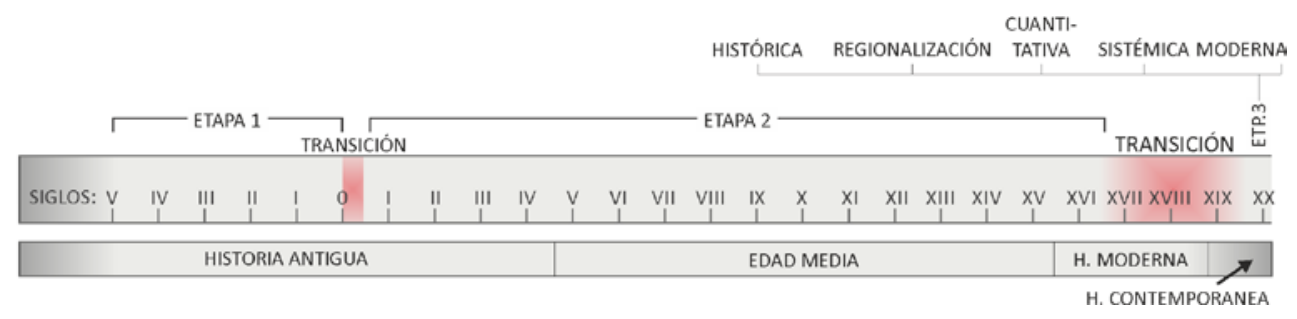

Esta interpretación de la Tierra desde una perspectiva secuencial (gradualismo) prosigue durante todo el siglo XIX, sobresaliendo la obra de Charles Lyell (Principles of Geology) publicada entre 1830 y 1833 en varios volúmenes. Tanto Lyell como Hutton pueden considerarse los padres de la geología moderna. Sus obras marcan un cambio en el contexto científico general de la segunda mitad del siglo XIX, sustituyéndose el idealismo por el positivismo, de modo que el único conocimiento válido es el científico/ empírico, desechándose cualquier tipo de tendencia inmaterial (ver Fig. 2). Es también en este periodo cuando surgen las primeras aportaciones de renombre netamente fluviales de la mano de geólogos estadounidenses como Powell (1875) y Dutton (1882) -desarrollando sus estudios en el Gran Cañón para comprender la acción erosiva de los ríos en la formación de valles y cauces-, el australiano Dana (1850) -quien indaga sobre la formación de los valles- o, de forma más notoria, Gilbert (1877) por sus aportaciones metodológicas (Gilbert, 1886) y concepción sistémica, en el sentido de que una acción (erosión) repercute sobre otra (deposición) y nada permanece estático sino en equilibrio dinámico. Términos tan recurrentes en la disciplina como "competencia" o "capacidad" forman también parte del rico legado de este autor. En el viejo continente, Nöe y Margerie (1888) en su tratado de las formas del terreno exaltan la escorrentía superficial como importante agente de modelado continental. 


\section{III.2. La Geomorfología Fluvial en el siglo XX}

En función de los grandes períodos históricos se podría decir, en un sentido laxo, que la geomorfología nace con la apertura de una tercera etapa circunscrita a la era contemporánea (3) (finales del siglo XIX - finales del siglo XX), erigiéndose como la fase de mayor desarrollo e importancia, tanto para la geomorfología en general como para la geomorfología fluvial en particular (Fig. 2). Con la corriente materialista instaurada de pleno en el seno de la sociedad de finales del siglo XIX, la producción científica comienza a crecer progresivamente, y la problemática se centra ahora únicamente en buscar una lógica a las expresiones naturales representadas en la faz de la Tierra. En función de la lectura de trabajos previos (Beckinsale y Chorley, 1991; Dollar, 2000, 2002, 2004), se considera como más apropiada la propuesta de Bauer (2004) de dividir en cinco fases esta tercera etapa histórica. La Fig. 2 trata de contextualizar cada una de estas etapas en su marco teórico, contexto filosófico y época histórica. Al mismo tiempo se señalan algunas obras que, en opinión de quien escribe, tuvieron mayor influencia en la geomorfología fluvial. Con la cita de estas obras no se pretende desmerecer a muchas otras no incluidas y que, tal vez, pudieran ser más relevantes que las aquí propuestas.

(3.1) Fase histórica (1890-1930): está dominada por la ideas del ciclo geográfico propuesto por William Morris Davis (Davis, 1899) para explicar la evolución del relieve. La obra The Geographical Cycle tuvo tal acogida científica que para muchos autores marca el nacimiento de la Geomorfología (Tricart, 1965). Esta repercusión mundial se debe, más que al contenido en sí, posteriormente cuestionado, a dos hechos (Gutiérrez, 2008): i) supone la primera metodología o paradigma propiamente geomorfológico; ii) aporta la primera interpretación evolutiva del relieve.

(3.2) Fase de regionalización (1920-1950): se caracteriza por la implantación de una metodología analítico-descriptiva que, englobando procesos y formas de la dinámica terrestre (Dickinson, 1976), permite estudiar minuciosamente cualquier región natural. Al tiempo que W.M. Davis desarrollaba su teoría sobre el ciclo geográfico, se llevaron a cabo investigaciones regionalistas en diversas partes del mundo (Richthofen, 1886; Nöe y Margerie, 1888; Penck, 1894; Passarge, 1912; Hettner, 1921), que documentaron sobre la variedad paisajística existente a nivel planetario. Esto ponía claramente en entredicho la simplicidad e inadecuación de la teoría del ciclo geográfico para alguno de estos lugares. Se comienza a forjar entonces una nueva tendencia en el estudio del relieve, que se concretiza en dos áreas bien definidas: un esbozo de la futura geomorfología dinámica, pero que en estos inicios presenta un enfoque muy estructural (Penck, 1894, 1905), centrado en el estudio cuantitativo de los procesos de los agentes modeladores y en la variabilidad temporal de las formas, aunque sin tener en consideración las combinaciones regionales; y la geomorfología climática (Büdel, 1948), que relaciona las formas del terreno con las zonas climáticas (Köppen, 1901). El postulado davisiano tuvo sus mayores detractores en la escuela germana, liderada primero por Albertch Penck y más tarde por su hijo Walter Penck, quienes defendían la componente estructural en la formación y evolución del relieve. Con todo, la diversidad de conjeturas respecto al ciclo geográfico y su indagación sobre la validez en otros territorios ha servido para ramificar el contenido de la geomorfología y, en consecuencia, enriquecerla con nuevos contenidos y experiencias que se fueron abriendo paso para acabar convirtiéndose en áreas especializadas. 
Figura 2

CRONOGRAMA HISTÓRICO DE LA GEOMORFOLOGÍA Y CONTEXTUALIZACIÓN DE ALGUNAS DE LAS OBRAS MÁS RELEVANTES PARA LA GEOMORFOLOGÍA FLUVIAL

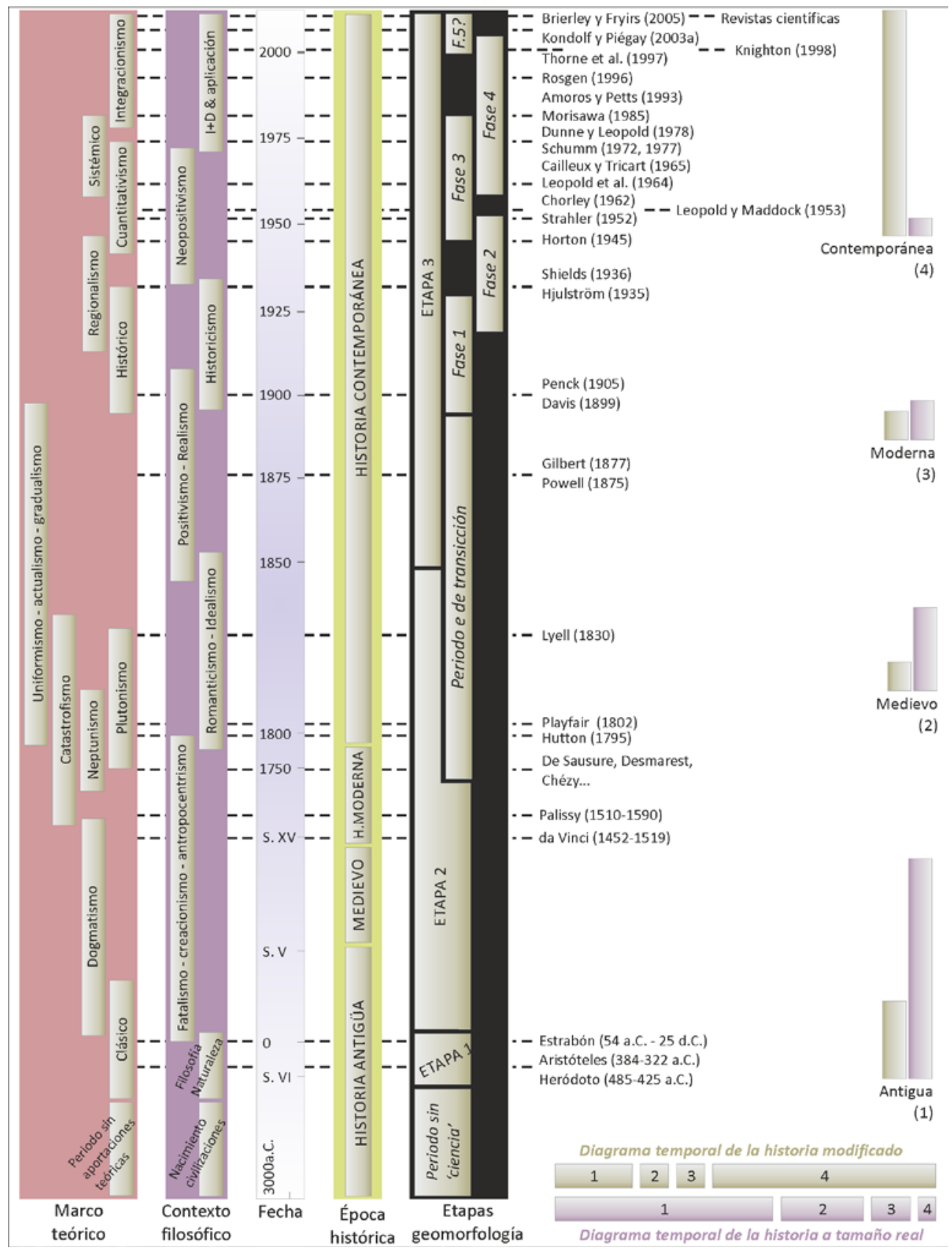


Durante este periodo se elaboraron interesantes manuales sobre geomorfología, algunos con base en las ideas cada vez más debilitadas de la teoría davisiana (Martonne, 1909), otros que tenían un enfoque también generalista pero críticos con el ciclo geográfico (Engeln, 1953; Thornbury, 1954). Igualmente, se llevaron a cabo trabajos adaptando el ciclo geográfico a diferentes medios del globo (Macar, 1946; Peltier, 1950; King, 1953). En cualquier caso, la visión regional, focalizada en una porción concreta de terreno, comienza a desvanecerse en detrimento de los análisis más amplios, muchos de ellos ya de carácter global.

(3.3) Fase cuantitativa (1940-1970): se trata de un periodo de metamorfosis suscitado por el formidable aporte de métodos y técnicas para la investigación en campo, laboratorio o gabinete (Cochran, 1953; Birot, 1955; Cailleux y Tricart, 1963; King, 1966; I.G.U., 1967; Doornkamp y King, 1971; Morisawa, 1976). La fase está encuadrada en un periodo con el neopositivismo o positivismo lógico como filosofía dominante y el reduccionismo como método (Fig. 2). Esto supuso una notable atomización de la disciplina, hecho que, a su vez, se tradujo en una pérdida de conexión entre sub-especialidades y en un distanciamiento con las ciencias madre geografía y geología (Bauer, 2004). Por el contrario, de modo inverso, se produce un acercamiento a otras disciplinas como la ingeniería hidráulica, edafología, biología, etc. En opinión de Pedraza (1996), el periodo cuantitativo, afín a otras ciencias de la Tierra, se caracteriza por: i) análisis sectorial de los procesos; ii) predominio de los estudios dinámicos (proceso) en detrimento de la forma y la evolución; iii) cuantificación de los procesos geomorfológicos; iv) uso de nuevas tecnologías y técnicas arrendadas a otras ciencias, especialmente a las matemáticas (marco empírico) y a la física (marco teórico) y con ello también a la ingeniería y v) definición de índices y parámetros que interpreten la morfología y dinámica del relieve.

Romero-Díaz y López-Bermúdez (1987) señalan que "los fundamentos de los estudios cuantitativos de morfometría fluvial hay que buscarlos en las experiencias y reflexiones científicas de Guglielmini (1967), Bernaoulli (1654-1705), Chezy (1718-1789), Manning, Brahms, y en las ideas de Hatton (1737-1824) quien enunció la ley de las "uniones" de los correspondientes tributarios, expresada a principios del siglo XIX por Playfair". A pioneros del movimiento cuantitativo como Gilbert (1914) -autor del primer estudio cuantitativo de las redes fluviales-, Sherman (1932), Hjulström (1935), Shields (1936) o Rubey (1938), les siguieron varios trabajos de fuerte impronta en el ámbito de la geomorfología fluvial: Horton (1932, 1945), cuya obra versó sobre redes de drenaje; Strahler (1952, 1964), quien se centró en la morfometría fluvial; Lane (1955) con valiosas aportaciones hidráulicas; Gumbel (1958) con su teoría estadística sobre los valores extremos; o Leopold y Maddock (1953) y Leopold et al. (1964) con contribuciones sobre la geometría hidráulica y los procesos fluviales en geomorfología, respectivamente. Las dimensiones científicas alcanzadas por estos autores, poniendo quizás mayor énfasis en los trabajos de Strahler (1951, 1954, 1956a, 1956b, 1964, 1968), Leopold y Maddock (1953) y Leopold et al. (1964), fue tal que marcaron la línea de trabajo en los años venideros, pero siendo actualmente todavía un referente.

A este respecto, Gregory (1976) fijó en siete las líneas de trabajo en geomorfología fluvial durante los años de contacto entre las tendencias cuantitativa y sistémica (fase 3.4): i) morfometría de la red de drenaje; ii) características de la cuenca de drenaje en relación a la producción de agua y sedimento; iii) investigación de la importancia de la dinámica en la generación de escorrentía; iv) relación de la morfología y los procesos con la geometría hidráulica del cauce; v) factores que controlan los modelos fluviales; vi) la paleohidrología y vii) enfo- 
ques teóricos del sistema fluvial. Con tal abanico de posibilidades se abre un periodo marcado por el creciente aumento en número y diversidad de publicaciones (Pardé, 1934; Mackin, 1948; Strahler, 1950; Schumm, 1956, 1972, 1977; Dylick, 1957; Dury, 1966; Chorley, 1967; Fairbridge, 1968; Morisawa, 1968, 1976). Es destacable el elevado número de manuales de geomorfología general que van surgiendo en diferentes países, por citar solamente algunos: Francia (Derruau, 1956; Birot, 1959), Brasil (Christofoletti, 1974) o Italia (Castiglioni, 1979).

(3.4) Fase de sistemas (1960-1980): esta fase es una consecuencia lógica del anterior periodo, en el sentido de que la pormenorizada aplicación matemático-estadística y la interpretación de la dinámica a una escala de tanto detalle (reduccionismo), resultan infructuosas para proporcionar tanto una visión general del comportamiento geomorfológico, como una evolución del paisaje (Bauer, 2004). Ya en 1952 es Strahler quien subraya la necesidad de que morfología y dinámica estén hiladas como un sistema dinámico y no como sistemas aislados que respondan al paradigma "causa-efecto" (Horton, 1932). El camino iniciado por Strahler lo continúan tres discípulos suyos de la Universidad de Columbia (Stanley Schumm, Richard Chorley y Mark Melton), siendo uno de ellos, Chorley (1962), el que introduzca la teoría general de sistemas (Bertalanffy, 1950; Hall y Fagen, 1956) en el conjunto de herramientas conceptuales de la geomorfología, con la cuenca fluvial como unidad geomorfológica básica (Leopold y Langbein, 1962; Chorley, 1969a; Gregory y Walling, 1973; Schumm, 1977).

La concepción sistémica de la geomorfología supuso que se adoptasen modelos teóricos de otras disciplinas científicas (física, matemáticas, biología...), lo cual contribuyó al enriquecimiento del léxico geomorfológico con cuantiosos términos (flujos de energía y masa, entropía, histéresis, estrés, umbral, etc.). Contrariamente, se produce una excesiva tendencia a parangonar los procesos geomorfológicos con un comportamiento sistémico, gran parte en detrimento de la investigación sobre procesos dinámicos (Bauer, 2004). También se levantan voces críticas contra la teoría de sistemas, asegurando que se trata de una aglomeración de conceptos e ideas más que de un arquetipo científico-experimental (Howard, 1965; Bauer, 2004; Gutiérrez, 2008). Si bien, en esta etapa se produce el despegue de la producción científica con un aumento progresivo de temáticas y, aunque en los primeros años dominan los aspectos sistémicos y cuantitativos, poco a poco va surgiendo una geomorfología más ambiental o aplicada (Tricart, 1953, 1962), altamente prolífica en EE.UU. (Chorley, 1969b; Leopold, 1974; Dunne y Leopold, 1978). De forma paralela emana una geomorfología teorética (Scheidegger, 1961; King, 1976; Chorley, 1978; Thornes, 1978) apoyada, a juicio del redactor, en cuatro conceptos clave para la compresión geomorfológica: magnitud y frecuencia (Wolman y Miller, 1960; Ritter, 1978) y escala temporal y espacial (Tricart, 1952; Schumm y Lichty, 1965; Chorley y Kennedy, 1971; Thornes y Brunsden, 1977, Cullingford et al., 1980; Thorn, 1982). Esta noción de tiempo y espacio resultó muy útil para contextualizar los diferentes trabajos de geomorfología, evitar auto-introspecciones y, en definitiva, cohesionar la disciplina (Bauer, 2004). El número de manuales de geomorfología también se extiende palmariamente (Sparks, 1972; Chorley et al., 1984), así como inquietudes por aspectos más ecológicos y de gestión (Oglesby et al., 1972; Whitton, 1975; Hails, 1977).

Resulta complicado enumerar todos y cada uno de los trabajos publicados en este periodo, pero de entre los mismos, se opina que son destacables por su contribución (al margen de los ya citados) las obras de: Chorley (1967), Allen (1970), Schumm (1972), Morisawa (1968, 1973, 1985), Gregory (1977), West (1978), Mather (1979), Schumm (1979), Thornes (1979), 
Strahler (1980). En el panorama editorial comienzan a surgir revistas especializadas como Journal of Hydrology (desde 1963); Water Resources Research (desde 1965); Zeitschrift fur Geomorphologie (desde 1921-1939; 1957); Revue de Géomorphologie Dynamique (desde 1952); Catena (desde 1973); Earth Surface and Processes (desde 1976); Progress in Physical Geography (desde 1977); Pysical Geography (desde 1980); Materialen zur Physiogeographie (desde 1980); Physio-Géo (desde 1981). En el ámbito nacional destaca, por ejemplo, Notes de Geografía Física (desde 1979).

(3.5) Fase moderna (1980-finales siglo XX): como postulaba Sala (1984: 229), "han aparecido una notable cantidad de textos dedicados a tratar tanto los presupuestos teóricos de la geomorfología dinámica como a estudiar a fondo los mecanismos y efectos de todos y cada uno de los grandes agentes geomorfológicos, (...) no sería de extrañar que en el futuro algunos de sus apartados llegaran a alcanzar suficiente entidad como para construir un tema independiente". En efecto, tres décadas después, las dimensiones que ha alcanzado la geomorfología fluvial la convierten en una asignatura independiente de numerosos programas de estudio, con un robusto corpus teórico y práctico. Esta quinta fase se corresponde con el tercer periodo secuencial que describe Gregory (2004) para la segunda mitad del pasado siglo. El autor estructura el periodo en tres fases o subperiodos, en donde el primero se define como un momento de importación y expansión, ligado al cuantitativismo; a este le sigue otro de consolidación, que vendría a ser el periodo entre la fase de sistemas y la fase moderna, donde se asientan las bases de la geomorfología actual; y un tercero de innovación, claramente encuadrado en el momento vigente.

La consumación actual de la geomorfología como una ciencia independiente está caracterizada por diferentes aspectos. El más significativo es la elevada especialización que experimenta, hecho común en la mayor parte de ciencias. El grado de detalle con que se abordan los estudios supone que, aun dentro de la propia disciplina, existan sub-disciplinas internas (Thorndycraft et al., 2008). Ahora bien, al tiempo que se produce una especialización de la geomorfología, esta también reafirma su identidad, gana en pragmatismo y se aleja de insustanciales discusiones sobre cuál es el verdadero paradigma. La expresión de esta tendencia es: i) el incremento de trabajos a escala de mucho detalle, descendiendo aquellos centrados en la evolución y la macrogeomorfología de grandes conjuntos y ii) con mayor interés por los procesos que por la forma (Barry, 1997). Valga como dato relevante que en los años ochenta el 75\% de las investigaciones en Gran Bretaña se ocupaban del estudio de los procesos a escala detallada (Gardner, 1983; citado en Gutiérrez, 2008).

En suma, se trata de una etapa con notables avances conceptuales, tecnológicos, prácticos y aplicados y, en consecuencia, prolija también en número de publicaciones. Muchas de las mismas están recogidas en trabajos que siguen la estela dejada por los clásicos manuales y enciclopedias geomorfológicas de los últimos lustros (Scheidegger, 1982, 1987; Summerfield, 1991; Ritter et al., 1995; Rhoads y Thorn, 1996; Stoddart, 1997; Huggett, 2003; Evans, 2004; Goudie, 2004; Gutiérrez, 2008). La producción en el ámbito fluvial continúa siendo pionera dentro del género geomorfológico con textos de miras amplias (Chorley et al., 1984; Morisawa y Clayton, 1985; Leopold, 1994; Knighton, 1998; Dollar, 2000, 2002, 2004; Martín Vide, 2006; Kondolf y Piégay, 2003a; Brierley y Fryirs, 2005; Schumm, 2005; Charlton, 2008), por otros orientados hacia aspectos más concretos (Richards, 1982; Zăvoianu, 1985; Petts y Foster, 1985; Thorne et al., 1987; Goudie, 1990; Amoros y Petts, 1993; Anderson et 
al., 1996; Rosgen, 1996; Tinkler y Wohl, 1998; Wohl, 2000; Bridge, 2003). No obstante, el campo de la ecología fluvial, y de forma derivada la geomorfología aplicada, es la que copa en los últimos años el mayor número de ejemplares (Verstappen, 1983; Cooke y Doornkamp, 1990; Newson, 1992; Thorne et al., 1997; Naiman y Bilby, 1998; Brizga y Finlayson, 2000; Anthony et al., 2001; Cushing y Allan, 2001; Schmitt, 2001; Allison, 2002; Sear et al., 2003; Downs y Gregory, 2004; Gordon, 2004; Brierley y Fryirs, 2005; Degoutte, 2006; Hauer y Lamberti, 2006; Newson y Large, 2006; Brierley y Fryirs, 2008; Harper y Ferguson, 2010; Malavoi y Bravard, 2010). En esta misma línea están los numerosos trabajos sobre restauración fluvial que comienzan a proliferar (González del Tánago y García de Jalón, 2007; Rinaldi et al., 2008; Booen y Raven, 2012; Simon et al., 2013). La acción antropogenética (Tricart, 1953) también está siendo objeto de abundantes investigaciones (Petts, 1984; Costa, 1995; Brandt, 2000), así como la aplicación de la geomorfología al análisis y mitigación de riesgos por crecidas e inundaciones (Wyzga, 1993, 1996; Díez Herrero, 2002; Benito, 2006; Benito et al., 2004; García Lorenzo, 2010; SNCZI, 2011).

El siglo XX, independientemente de las fases históricas en que este se pueda dividir, ha supuesto la auténtica expansión de la geomorfología fluvial, en especial en la segunda mitad del siglo XX. Así, a partir de la década de 1960 comienza a darse un notable incremento en el número de artículos de revistas científicas. Sin embargo, será de 1970/80 en adelante cuando se produzca el verdadero boom con el nacimiento y propagación de artículos científicos en numerosas revistas (Kondolf y Piégay, 2003b). Este fenómeno no deja de ser más que un escaparate de la alta diversidad experimentada por la ciencia. Sobresalen algunas revistas de gran prestigio como Water Research, The Geological Society of American Bulletin o Progress in Physical Geography (Tabla 2), por otras de corto recorrido como Regulated Rivers: Research and Management (1984-2001), River Research and Applications (desde 2001) o, por ejemplo, Aquatic Conservation: Marine and Freshwater Ecosystems (desde 1990).

Tabla 2

CARACTERISTIICAS DE IMPACTO DE ALGUNAS DE LAS PRINCIPALES REVISTAS EN GEOMORFOLOGÍA FLUVIAL PARA EL PERIODO 2013/2014. LOS DATOS FUERON OBTENIDOS EL 22/01/2015 DESDE LA WEB WWW.BIOXBIO.COM, DONDE FI ES EL FACTOR DE IMPACTO, ARTÍCULOS EL NÚMERO DE ESTOS PUBLICADOS Y CITAS EL NÚMERO TOTAL DE VECES QUE ELARTÍCULO FUE CITADO

\begin{tabular}{|l|l|c|c|c|}
\hline \multicolumn{1}{|c|}{ Título } & \multicolumn{1}{c|}{ País } & FI & Artículos & Citas \\
\hline Water Research & England & 5,323 & 675 & 49.606 \\
\hline The Geological Society of America Bulletin & USA & 4,398 & 102 & 14.523 \\
\hline Progress in Physical Geography & England & 3,885 & 42 & 2.488 \\
\hline Water Resource Research & USA & 3,709 & 616 & 34.109 \\
\hline Hydrological Processes & USA & 2,696 & 321 & 13.639 \\
\hline Earth Surface Processes and Landforms & England & 2,695 & 159 & 7.114 \\
\hline Journal of Hydrology & Netherlands & 2,693 & 627 & 29.080 \\
\hline Geomorphology & Netherlands & 2,577 & 323 & 11.912 \\
\hline Catena & Germany & 2,482 & 193 & 5.799 \\
\hline
\end{tabular}


En esta misma línea se comienzan a fraguar en numerosos países asociaciones y grupos de investigación nacionales e internacionales (por destacar algunos): The British Society for Geomorphology (Reino Unido), Associazione Italiana di Geografia Fisica e Geomorfologia (Italia), The Japanese Geomorphological Union (Japón), Groupe Français de Géomorphologie (Francia), The Geographical Society of China (China), The Geomorphology Specialty Group of the Association of American Geographers (EE.UU.), The Southern African Association of Geomorphologists (Sudáfrica), Mexican Society of Geomorphology (México), Uniao da Geomorfologia Brasileira (Brasil), Australia New Zealand Geomorphology Group (Australia y Nueva Zelanda), The Canadian Geomorphology Research Group (Canadá), Deutscher Arbeitskreis für Geomorphologie (Alemania), Sociedad Española de Geomorfología (España). Todas estas asociaciones nacionales tienen en la International Association of Geomorphologists un punto común de encuentro. La parte fluvial de estos grupos generales de geomorfología es minoritaria, siendo dominantes los riesgos geomorfológicos (en donde los riesgos relacionados con ambientes fluviales compiten con más riesgos).

\section{III.3. La Geomorfología Fluvial en el siglo XXI}

El hecho más relevante de la actual geomorfología fluvial respecto a la de décadas anteriores es el uso masivo de las nuevas tecnologías (Murray et al., 2009; Micheletti et al., 2014; Tarolli, 2014; Woodget et al., 2014). Stott (2010) desarrolló un informe sobre el estado de la disciplina durante los años 2006/07 a través del análisis de 147 artículos publicados en 21 revistas de peso. Los resultados del estudio indican que son 10 las áreas temáticas de mayor investigación dentro de la geomorfología fluvial actual: gestión fluvial, restauración y efectos de la vegetación sobre los sistemas fluviales, erosión del suelo, hidráulica fluvial, transporte de sedimentos, transferencia de sedimentos en ladera, modelización del medioambiente fluvial, regulación de ríos, cambios en el cauce e influencia humana, avances en metodologías en geomorfología fluvial, erosión de orillas y estudios paleoambientales.

Este cambio de tendencia de la geomorfología fluvial, pasándose a una nueva era liderada por las nuevas tecnologías (Murray et al., 2009), es motivo de debate y reflexión para la comunidad científica. Por una parte se discuten los valores que se pueden perder con la nueva tendencia. Pero, por otra parte, surgen numerosos trabajos que enfatizan en las virtudes que las tecnologías traen consigo. Asimismo, también aparecen estudios que tratan de hacer una recopilación, a modo de cierre de etapa, de la tradición geomorfológica de los últimos años (Vogel, 2011; Butler, 2013; Wohl, 2013). Esta nueva etapa de uso de las nuevas tecnologías (fase 5 de la Fig. 2) establece un cambio notable en las formas de proceder, analizar y obtener resultados. Como señala Vitek (2013), las formas y procesos en geomorfología del siglo XIX poco tienen en común con las desarrolladas en el siglo XXI, si bien, aun dentro de un intervalo de tiempo tan corto como lo son los 15 años de siglo XXI, los cambios producidos en los métodos de las ciencias de la tierra son muy elevados (Murray et al., 2009; Vitek, 2013). Pero estos cambios no son únicamente grandes en contenido y formas, sino que también se caracterizan por ser altamente cambiantes y dinámicos, con lo que es posible que en los próximos años haya que hablar de nuevos avances que irán moldeando el método geomorfológico (Vitek, 2013; Church, 2013). Esta línea de cambio de la geomorfología fluvial fue ya analizada e intuida en la década de los años 90 por diversos estudios (Rhoads, 1994; Rhoads y Thorn, 1993, 1994, 1996). 
El trabajo de Harden (2013) también ofrece una interesante visión del contexto de la geomorfología hoy en día. La autora pone de relieve uno de los aspectos que pueden dar pie a mayor debate dentro de la nueva etapa tecnológica: “trabajo de campo desde gabinete?”. No cabe duda que las nuevas tecnologías ofrecen posibilidades de análisis más amplias, lo que conlleva a extraer conclusiones más generalistas, no tan reduccionistas, y así apreciar la riqueza y complejidad de los sistemas fluviales (Harden, 2013). Parece incuestionable que el trabajo de campo en geomorfología debe ser algo consustancial al propio trabajo de gabinete, a pesar de las cada vez mayores posibilidades que ofrece este último. Esta problemática también ha sido objeto de debate en el simposio The Field Tradition in Geomorphology 43rd Annual Binghamton Geomorphology, celebrado el 21-23 de septiembre de 2012 en Jackson, Wyoming (USA).

En lo que respecta a la geomorfología fluvial en España el comportamiento es muy diferente, tanto internamente como comparándolo con otros países. En el trabajo de García Ruiz $(1999,2008)$ sobre el impacto y producción científica de la geomorfología española, se extrae que la revista Cuaternario y Geomorfología experimentó un notable incremento en el número de artículos en las dos últimas décadas y algunos de estos se encuentran entre los más citados de la Geomorfología española. No obstante, el número de trabajos dedicados a la geomorfología dinámica en ambientes fluviales sigue siendo muy escaso. Y ello a pesar de que el desarrollo actual de la geomorfología fluvial española es muy destacable, apoyándose en grupos de investigación muy activos que se dedican a temáticas diversas. En los Pirineos y Cataluña se trabaja especialmente en el análisis de procesos y en las consecuencias de la regulación hidrológica en los cauces (García Ruiz et al., 2010, 2011; Sanjuán et al., 2014; Batalla et al., 2004; Vericat y Batalla, 2010; Batalla y Vericat, 2011, 2013; Batalla et al., 2014), incluyendo aportaciones de ingenieros fluviales (Martín Vide y Andreatta (2008), Martín Vide et al., 2010; Ferrer-Boix, 2010). En Valencia y Murcia hay valiosas aportaciones al estudio de las ramblas mediterráneas (Mateu, 2000; Segura y Sanchis, 2013; Conesa y García Lorenzo, 2009) y en Andalucía a la dinámica fluvial asociada a elementos históricos y usos del suelo (Guerrero y Baena, 2002; García Martínez et al., 2009). Desde el ámbito de la geología destacan los trabajos sobre ríos en roca (Ortega y Garzón, 2009; Ortega y Durán, 2010; Ortega et al., 2014), las paleocrecidas (Benito y Thorndycraft, 2005), así como los análisis y cartografías geomorfológicas aplicadas a la gestión de riesgos desde organismos como el IGME (Díez Herrero et al., 2008, 2013; Lastra et al., 2008), el INDUROT (Sánchez Martínez y Lastra, 2011) y la universidad (Uribelarrea, 2008). Por último, el grupo que desarrolla su investigación en Aragón, País Vasco y Galicia está trabajando en clasificación fluvial, diagnóstico hidromorfológico y aplicaciones a la restauración fluvial y a la gestión de riesgos (Ollero, 2010; Ollero et al., 2011, 2014; Ibisate et al., 2013; Ibisate, 2014; Horacio y Ollero, 2011; Horacio, 2012, 2014).

\section{REFLEXIONES FINALES}

La evolución histórica de la geomorfología fluvial aquí presentada no tiene conclusiones propiamente dichas. Un proceso histórico es el que es, aunque pudiera ser discutible la forma de abordar este estudio o las fuentes de datos e interpretación que de estas se hicieron. Sin embargo, lo que parece incuestionable es que los acontecimientos históricos multicausales que se han ido sucediendo en el tiempo son de gran utilidad para alcanzar una comprensión lógica de lo que actualmente es la geomorfología fluvial. 
La mirada trazada hacia el pasado y presente de la geomorfología fluvial invita a pensar en un futuro en donde esta tenga una presencia cada vez más robusta en los planes de gestión y buenas prácticas fluviales. Pero esta buena praxis solo es posible si la geomorfología gana enteros en los presupuestos que los gobiernos dedican a investigación. En este sentido, no hay lugar a dudas de que, por ejemplo, una restauración fluvial no puede concebirse sin la geomorfología, del mismo modo que la admiración y valoración de la sociedad por los ríos es mayor si se conocen sus mecanismos de funcionamiento.

Las líneas que se considera tendrán mayor pujanza en los próximos años dentro de la geomorfología fluvial podrían seguir un triple camino. Cualquiera de las vías presenta un marcado corte analítico-científico pero con vocación aplicable, y no solo desde el ámbito científico sino también del gestor. La primera de estas tres líneas sería la aplicación de las nuevas tecnologías en la obtención de datos cuantitativos (Wheaton, 2008; Westoby et al., 2012; Woodget et al., 2014). Una segunda línea de actuación apunta hacía la mejora en la aplicación de modelos. Se trataría de enfatizar más en técnicas que automaticen procesos y ayuden en la interpretación geomorfológica (Alber y Piégay, 2011). El tercer campo de acción se focaliza en la restauración fluvial. Un proceso de restauración es un proceso del que nunca se sabe lo suficiente porque cada caso, cada río, cada tramo y cada momento es diferente de otro. Esto supone una complejidad añadida a la que ya de por sí tiene la restauración fluvial. La única forma de paliar dicha complejidad es profundizar en el conocimiento de las otras dos líneas de investigación descritas

La rápida y sólida irrupción de internet y las redes sociales en las sociedades avanzadas también ha supuesto un nuevo enfoque para la geomorfología fluvial. El concepto sociológico que aportan las redes sociales, en su afán por compartir información, ha servido para que la palabra "geomorfología" saliese del ostracismo al que estuvo sometida durante muchos años. Esta revolución está marcando un cambio lento pero constante en la introducción a la ciudadanía de la figura del geomorfólogo/a. Se podría decir que estamos en la era de la geomorfología fluvial 3.0.

\section{BIBLIOGRAFÍA}

ALBER, A. y PIÉGAY, H. (2011): «Spatial disaggregation and aggregation procedures for characterizing fluvial features at the network-scale: Application to the Rhône basin (France)». Geomorphology, n 125 (3), 343-360.

ALLEN, J.R.L. (1970): Physical processes of sedimentation. Londres. Allen \& Unwin.

ALLISON, R.J. (2002): Applied geomorphology. Theory and practice. Chichester. John Wiley \& Sons.

AMOROS, C. y PETTS, G.E. (1993): Hydrosystèmes fluviaux. Paris. Masson.

ANDERSON, M.G., WALLING, D.E. y BATES, P.D. (1996): Floodplain processes. Chichester. John Wiley \& Sons.

ANTHONY, D.J., HARVEY, M.D., LARONNE, J.B. y MOSLEY, M.P. (2001): Applying Geomorphology to environmental management. Denver, Colorado, USA. Water Resources Publication.

BARRY, R.G. (1997): «Palaeoclimatology, climate system processes and the geomorphic record» in Process and Form in Geomorphology (Stoddart, D.R., Ed.). London, Routledge, 187-214. 
BATALLA, R.J. y VERICAT, D. (2011): «An appraisal of the contemporary sediment yield in the Ebro Basin». Journal of Soils and Sediments, $\mathrm{n}^{\circ} 11,1.070-1.081$.

BATALLA, R.J. y VERICAT, D. (2013): «Rivers architecture supporting life» in River conservation: challenges and opoortunities (Sabater, S. y Elosegi, A., Eds.). Madrid, Fundación BBVA, 61-76.

BATALLA, R.J., GÓMEZ, C.M. y KONDOLF, G.M. (2004): «Reservoir-induced hydrological changes in the Ebro river basin (NE Spain)». Journal of Hydrology, n 290, 117-136.

BATALLA, R.J., VERICAT, D. y TENA, A. (2014): «The fluvial geomorphology of the lower Ebro (2002-2013): bridging gaps between management and research». Cuadernos de Investigación Geográfica, $\mathrm{n}^{\circ} 40$ (1), 29-52.

BAUER, B.O. (2004): «Geomorphology» in Ecyclopedia of Geomorphology (Goudie, A.S., Ed.). London, Routledge, 428-435.

BECKINSALE, R.P. y CHORLEY, R.J. (1991): The History of the Study of Landforms or the Development of Geomorphology. Historical and Regional Geomorphology 1890-1950. New York. Routledge.

BENITO, G. (2006): «Riesgos de inundaciones. Tendencias históricas y perspectivas de acuerdo con el cambio climático». Cuaternario y Geomorfología, no 20 (3-4), 29-44.

BENITO, G. y THORNDYCRAFT, V.R. (2005): «Palaeoflood hydrology and its role in applied hydrological sciences». Journal of Hydrology, $\mathrm{n}^{\circ}$ 313, 3-15.

BENITO, G., DÍEZ-HERRERO, A. y FERNÁNDEZ DE VILLALTA, M. (2004): «Flood response to NAO and Solar Activity in the Tagus Basin (Cental Spain) over the last millennium». Climatic Change, $\mathrm{n}^{\circ}$ 66, 27-28.

BERTALANFFY, VON A. (1950): «Theory of open systems in physics and biology». Science, $\mathrm{n}^{\circ} 111,23-29$.

BIROT, P. (1955): Les méthodes de la morphologie. Paris. Presses Universitaries de France. BIROT, P. (1959): Precis de Géographie Physique Generale. Paris. Lib. Armand Colin.

BOON, P.J. y RAVEN, P.J. (2012): River conservation and management. Chichester. WileyBlackwell.

BRANDT, S.A. (2000): «Prediction of downstream geomorphological changes after dam construction: a stream power approach». Water Resources Development, no 16 (3), 343-367.

BRIDGE, J.S. (2003): Rivers and floodplains. Forms, processes, and sedimentary record. Oxford. Blackwell Science.

BRIERLEY, G.J. y FRYIRS, K.A. (2005): Geomorphology and river management. Applications of the river styles framework. Oxford. Blackwell.

BRIERLEY, G.J. y FRYIRS, K.A. (2008): River futures. An integrative scientific approach to river repair. Washington, Island Press.

BRIZGA, S. y FINLAYSON, B. (2000): River management. The Australian experience. Chichester. John Wiley \& Sons.

BÜDEL, L. (1948): «Das system der klimatischen Geomorphologie (Beiträge zur Geomorphologie der Klimazonen und Vorzeitklimate V.)». Verhandlungen Deutscher Geographentag. Munchen, $\mathrm{n}^{\circ} 27,65-100$.

BUTLER, D.R. (2013): «The field tradition in mountain geomorphology». Geomorphology, $\mathrm{n}^{\circ} 200,42-49$. 
CAILlEUX, A. y TRICART, J. (1963): Initiation B l'étude de sables et de galets. Paris, CDU.

CAPEL, H. (1983): «Positivismo y antipositivismo en la ciencia geográfica. El ejemplo de la geomorfología». GeoCrítica, n ${ }^{\circ}$ 33, 1-53.

CASTIGLIONI, G.B. (1979): Geomorfologia. Torino. UTET.

CHARLTON, R. (2008): Fundamentals of fluvial geomorphology. London and New York. Routledge.

CHORLEY, J. (1978): «Bases for theory in geomorphology» in Geomorphology. Present problems and future prospects (Embleton, C., Brunsden, D. y Jones, D.K.C., Eds.). John Wiley \& Sons, Oxford, 1-13.

CHORLEY, R.J. (1962): Geomorphology and General System Theory. USA. U.S. Geol. Surv. Prof. Paper.

CHORLEY, R.J. (1967): «Models in geomorphology in Models» in Geography (Chorley, R.J. y Hagget, P., Eds.). Methuen, 59-96.

CHORLEY, R.J. (1969a): «The drainage basin as the fundamental geomorphic unit» in Water, Earth and Man (Chorley, R.J., Ed.). London, Methuen and Co. Ltd., 77-100.

CHORLEY, R.J. (1969b): Water, Earth and Man. A Synthesis of Hydrology, Geomorphology and Socio-Economic Geography. London. Methuen and Co. Ltd.

CHORLEY, R.J. y KENNEDY, A.B. (1971): Physical geography. A systems approach. London. Prentice-Hall.

CHORLEY, R.J., SCHUMM, S.A. y SUGDEN, D.E. (1984): Geomorphology. Earth Sciences and the Past. London. Methuen and Co. Ltd.

CHRISTOFOLETTI, A. (1974): Geomorfologia. Edgard Blucher.

CHURCH, M. (2013): «Refocusing geomorphology. Field work in four acts». Geomorpho$\log y, \mathrm{n}^{\circ} 200,184-192$.

COCHRAN, W.G. (1953): Sampling technique. New York. John Wiley \& Sons.

CONESA, C. y GARCÍA LORENZO, R. (2009): «Bed texture changes caused by check dams on ephemeral channels in mediterranean semiarid environments». Zeitschrift für Geomorphologie, $\mathrm{n}^{\circ} 52$ (4), 437-461.

COOKE, R.U. y DOORNKAMP, J.C. (1990): Geomorphology in environmental management. A new introduction. Oxford. Clarendon Press.

COSTA, J.E. (1995): Natural and anthropogenic influences in fluvial geomorphology. USA. American Geophysical Union.

CULLINGFORD, R.A., DAVIDSON, D.A. y LEWIN, J. (1980): Timescales in geomorphology. New York. John Wiley \& Sons.

CUSHING, C.E. Y ALLAN, J.D. (2001): Streams. Their ecology and life. San Diego. Academic Press.

DANA, J.D. (1850): «On the degradation of the rocks of New South Wales and formation of valleys». Am. J. Sci., no 2(9), 289-294.

DAVIS, W.M. (1899): «The Geographical Cycle». Geographical Journal, nº 14, 481-504.

DEGOUTTE, G. (2006): Diagnostic, aménagement et gestion des rivières. Hydraulique et morphologie fluviales appliquées. Paris. Tec and Doc Lavoisier.

DERRUAU, M. (1956): Précis de Géomorphologie. Paris. Masson. 
DICKINSON, R.E. (1976): Regional concept. The anglo-american leaders. Londres. Routledge and Kegan Paul.

DÍEZ HERRERO, A., BALLESTEROS, J.A., RUIZ VILLANUEVA, V. y BODOQUE, J.M. (2013): «A review of dendrogeomorphological research applied to flood risk analysis in Spain». Geomorphology, no 196, 211-220.

DÍEZ HERRERO, A., LAÍN, L. y LLORENTE, M. (2008): Mapas de peligrosidad por avenidas e inundaciones. Guía metodológica para su elaboración. Madrid. Instituto Geológico y Minero de España.

DÍEZ-HERRERO, A. (2002): «Aplicaciones de los Sistemas de Información Geográfica al análisis del riesgo de inundaciones fluviales» en Los Sistemas de Información Geográfica en la gestión de los riesgos geológicos y el medio ambiente (Laín Huerta, L., Ed.). Madrid, Instituto Geológico y Minero de España. Serie. Medio Ambiente. Riesgos Geológicos, 87-112.

DOLLAR, E.S.J. (2000): «Fluvial geomorphology». Progress in Physical Geography, n 24, 385-406.

DOLLAR, E.S.J. (2002): «Fluvial geomorphology». Progress in Physical Geography, n 26, 123-143.

DOLLAR, E.S.J. (2004): «Fluvial geomorphology». Progress in Physical Geography, n 28, 405-450.

DOORNKAMP, J.C. y KING, C.A.M. (1971): Numerical analysis in geomorphology. London. Edward Arnold.

DOWNS, P.W. y GREGORY, K.J. (2004): River channel management. Towards sustainable catchment hydrosystems. London. Edward Arnold.

DUNNE, T. y LEOPOLD, L.B. (1978): Water in environmental planning. San Francisco. Freeman and Co.

DURY, G.H. (1966): Essays in geomorphology. London. Heinemann.

DUTTON, C.E. (1882): The Physical Geology of the Grand Cañon. Washington. Government Printing Office.

DYLICK, J. (1957): «Dynamical geomorphology, its nature and methods». Bull. Soc. SC. et Lett., Lodz, 111-VIII, 12, 1-42.

ENGELN, O.D. VON (1953): Geomorphology. Systematic and regional. New York. Macmillam.

EVANS, D.J.A. (2004): Geomorphology. London. Routledge.

FAIRBRIDGE, R.W. (1968): The Encyclopedia of Geomorphology. Stroudsburg. Dowden, Hutchinson and Ross.

FERRER-BOIX, C. (2010): Incisión de ríos por extracción aluvial y retirada de presas. Estudio matemático y experimental. Tesis doctoral, Universitat Politècnica de Catalunya, Barcelona.

GARCÍA LORENZO, R. (2010): Peligrosidad de las aguas de avenidas en los cruces de carreteras con ramblas. Estudio aplicado a la franja costera meridional de la región de Murcia. Tesis doctoral, Universidad de Murcia, Murcia.

GARCÍA MARTÍNEZ, B., BAENA, R. y POSADA, C. (2009): «La acción antrópica como inductora de cambios en el patrón fluvial del río Rivera de Huelva (sector Guillena-Sevilla)» en Avances en estudios sobre desertificación: aportaciones al Congreso Interna- 
cional sobre Desertificación en memoria del profesor John B. Thornes (Romero, M.A., Belmonte, F. Alonso, F. López Bermúdez, F., Eds.). Murcia, Universidad de Murcia, 473-476.

GARCÍA RUIZ, J.M. (1999): La producción científica de la Geomorfología española y su impacto, a través de las publicaciones periódicas. Zaragoza, Instituto Pirenaico de Ecología.

GARCÍA RUIZ, J.M. (2008): «Cuaternario y Geomorfología en la literatura internacional». Rev. C. \& G., n 22 (1-2), 11-16.

GARCÍA RUIZ, J.M. y LANA-RENAULT, N. (2011): «Hydrological and erosive consequences of farmland abandonment in Europe, with special reference to the Mediterranean region. A review». Agriculture, Ecosystems and Environment, $\mathrm{n}^{\circ}$ 140, 317-338.

GARCÍA RUIZ, J.M., LANA-RENAULT, N., BEGUERÍA, S., LASANTA, T., REGÜÉS, D., NADAL, E., SERRANO, P., LÓPEZ MORENO, J.I., ALVERA, B., MARTÍ BONO, C. y ALATORRE, L.C. (2010): «From plot to regional scales: interactions of slope and catchment hydrological and geomorphic processes in the Spanish Pyrenees». Geomorphology, $\mathrm{n}^{\mathrm{o}}$ 120, 248-257.

GARDNER, R. (1983): «Introduction» in Mega-Geomorphology (Gardner, R. y Scoging, H., Eds). Oxford, Clarendon Press.

GEACH, M.R., STOKES, M., TELFER, M.W., MATHER, A.E., FYFE, R.M. y LEWIN, S. (2014): The application of geospatial interpolation methods in the reconstruction of Quaternary landform records. Geomorphology, 216, 234-246.

GEORGE, P. (2007): Diccionario Akal de Geografía. Madrid. Akal.

GILBERT, G.K. (1877): Report on the Geology of the Henry Mountains. Washington. U.S. Geographical and Geological Survey of the Rock Mountain Region.

GILBERT, G.K. (1886): «The inculcation of the scientific method by exemple». Amer. Jour. Sci. 3rd Ser., no 31, 284-299.

GILBERT, G.K. (1914): The transportation of debris by running water. USA. United States Geological Survey, Professional Paper.

GLACKEN, C.J. (1996): Huellas en la playa de Rodas. Naturaleza y cultura en el pensamiento occidental desde la Antigüedad hasta finales del siglo XVIII. Ed. del Serbal (ed. española, original de 1967).

GONZÁLEZ DEL TÁNAGO, M. y GARCÍA DE JALÓN, D. (2007): Restauración de ríos. Guía metodológica para la elaboración de proyectos. Madrid. Ministerio de Medio Ambiente.

GORDON, N.D. (2004): Stream hydrology. An introduction for ecologists. Chichester. John Wiley \& Sons.

GOUDIE, A. (1990): Geomorphological techniques. London. Routledge.

GOUDIE, A.S. (2004): Ecyclopedia of Geomorphology. London. Routledge.

GRAU, R. y SALA, M. (1982): «La geomorfología en sus tratados y manuales. Un esquema histórico de la disciplina (1870-1982)». Revista de Geografía, n XVI-XVII, 175-192.

GREGORY, K.J. (1976): «Changing drainage basins». Geographical Journal, nº 142, 237 247.

GREGORY, K.J. (1977): River channel changes. Chichester: Wiley. 
GREGORY, K.J. (2004): «Fluvial geomorphology» in Ecyclopedia of Geomorphology (Goudie, A.S., Ed.). London, Routledge, 392-398.

GREGORY, K.J. y WALLING D.E. (1973): Drainage basin form and process. London. Edward Arnold.

GUERRERO, I. y BAENA, R. (2002): «Geomorfología fluvial y restauración ambiental: el ejemplo del río Guadiamar en la zona de Entremuros (Parque Natural de Doñana)». Estudios recientes (2000-2002) en Geomorfología, Patrimonio, montaña y dinámica territorial. Valladolid, 79-90.

GUMBEL, F.J. (1958): Statistics of Extremes. New York, Columbia University Press.

GUTIÉRREZ, M. (2008): Geomorfología. Madrid. Pearson Educación.

HAILS, J.R. (1977): Applied geomorphology. A perspective of the contribution of geomorphology to interdisciplinary studies and environmental management. Amsterdam. Elsevier.

HALL, A.D. y R.E. FAGEN (1956): Definition of System. General Systems (Yearbook of the Society for the Advancement of General Systems Theory), $\mathrm{n}^{\circ} 1,18-28$.

HARDEN, C.P. (2013): «Geomorphology in context. Dispatches from the field». Geomorphology, $\mathrm{n}^{\circ}$ 200, 34-41.

HARPER, D.M. y FERGUSON, A.J.D. (2010): The ecological basis for river management. Chichester. Wiley.

HART, M.G. (1986): Geomorphology pure and applied. London. Allen and Unwin.

HAUER, F.R. y LAMBERTI, G.A. (2006): Methods in stream ecology. New York. Academic Press.

HETTNER, A. (1921): Die Oberflächenformen des Festlandes. Stuttgart. Springer Fachmedien Wiesbaden.

HJULSTRÖM, F. (1935): «Studies of the morphological activity of rivers as illustrated by River Fyris». Bulletin of the Geological Institute, Uppsala, vol. XXV, n 3, 221-527 pp.

HORACIO, J. (2012): «Caracterización geomorfológica de los ríos de Galicia mediante unidades litotopográficas». Geographicalia, nº 62, 35-66.

HORACIO, J. (2014): Geomorfología fluvial en sistemas atlánticos: metodología de caracterización, clasificación y restauración para los ríos de Galicia. Tesis doctoral, Universidade de Santiago de Compostela, Santiago de Compostela.

HORACIO, J. y OLLERO, A. (2011): «Clasificación geomorfológica de cursos fluviales a partir de Sistemas de Información Geográfica (S.I.G.)». Boletín de la Asociación de Geógrafos Españoles, n⿳0 56, 373-396.

HORTON, R.E. (1932): «Drainage basin characteristics». Transactions American Geophysical Union, $\mathrm{n}^{\mathrm{o}} 13,350-361$.

HORTON, R.E. (1945): «Erosional development of streams and their drainage basins. Hydrophysical approach to quantitative morphology». Bulletin of the Geologial Society of America, $\mathrm{n}^{\circ}$ 56, 275-370.

HOWARD, A.D. (1965): «Geomorphic systems, equilibrium and dynamics». Arn. J. Sci., no 263, 302-312.

HUGGETT, R.J. (2003): Fundamentals of geomorphology. London. Routledge.

HUTTON, J. (1795): Theory of the Earth. Edimburgh. William Creek edit. 
IBISATE, A., DÍAZ, E., OLLERO, A., ACÍN, V. y GRANADO, D. (2013): «Channel response to multiple damming in a meandering river, middle and lower Aragón River (Spain)». Hydrobiologia, $\mathrm{n}^{\circ}$ 712, 5-23.

INTERNATIONAL GEOGRAPHICAL UNION (I.G.U.) (1967): «Field methods for the study of slope and fluvial processes. A contribution to the International Hydrological Decade». Revue de géomorphologie dynamique, $\mathrm{n}^{\circ}$ 17(4), $44 \mathrm{pp}$.

KING, C.A.M. (1966): Techniques in Geomorphology. London. Arnold.

KING, C.A.M. (Ed.) (1976): Landforms and geomorphology. concepts and history. Stroudsburg, Pennsylvania. Dowden, Hutchinson and Ross.

KING, L.C. (1953): «Canons of landscape evolution». Bull. Geol. Soc. America, n 64, 721-752.

KNIGHTON, D. (1998): Fluvial forms and processes. A new perspective. London. Arnold.

KONDOLF, G.M. y PIÉGAY, H. (2003a): Tools in Fluvial Geomorphology. Chichester. Wiley.

KONDOLF, G.M. y PIÉGAY, H. (2003b): «Tools in fluvial geomorphology. Problem statement and recent practice» in Tools in Fluvial Geomorphology (Kondolf, G.M. y Piégay, H., Eds.). Chichester, Wiley, 3-22.

KÖPPEN, W. (1901): Versuch einer Klassification der Klimate vorzugsweise nach ihren Beziehungen zur Pflanzenwelt. Geogr. Z.

LANE, E.W. (1955): «Design of stable channels». Transactions of the American Society of Civil Engineers, $\mathrm{n}^{\circ} 120,1.234-1.260$.

LASTRA, J., FERNÁNDEZ, E., DÍEZ HERRERO, A. y MARQUÍNEZ, J. (2008): «Flood hazard delineation combining geomorphological and hydrological methods: an example in the Northern Iberian Peninsula». Natural Hazards, $n^{\circ} 45,277-293$.

LEOPOLD, L.B. (1974): Water, a primer. San Francisco. Freeman.

LEOPOLD, L.B. (1994): A view of the river. Harvard University Press.

LEOPOLD, L.B. y LANGBEIN, G.W. (1962): The concept of entropy in landscape evolution. U.S. Geol. Surv. Prof. Paper, 500-A.

LEOPOLD, L.B. y MADDOCK, T. (1953): The hydraulic geometry of stream channels and some physiographic implications. Washington. U. S. Geological Survey Prof. paper.

LEOPOLD, L.B., WOLMAN, M.G. y MILLER, J.P. (1964): Fluvial processes in Geomorphology. San Francisco. Freeman and Co.

LYELL, C. (1833): Principles of geology. London John Murray, Albemarle Street.

MACAR, P. (1946): Principles de géomorphologie normale, étude des formes du terrain des régions d climat humide. Liège. Masson.

MACKIN, J.H. (1948): «The concept of the graded river». Geological Society of American Bulletin, $\mathrm{n}^{\circ}$ 59, 463-512.

MALAVOI, J.R. y BRAVARD, J.P. (2010): Éléments d'hydromorphologie fluviale. Vincennes. Office National de l'Eau et des Milieux Aquatiques (ONEMA).

MARTÍN VIDE, J.P. (2006): Ingeniería de ríos. Barcelona. Edicions UPC.

MARTÍN VIDE, J.P. y ANDREATTA, A. (2008): «Channel degradation and slope adjustment in steep streams controlled through bed sills». Earth Surface Processes and Landforms, $n^{\circ} 34,38-47$. 
MARTÍN VIDE, J.P., FERRER-BOIX, C. y OLLERO, A. (2010): «Incision due to gravel mining: modeling a case study from the Gállego River, Spain». Geomorphology, nº 117, 261-271.

MARTONNE, E. DE (1909): Traité de géographie physique. Paris. A. Colin.

MATEU, J.F. (2000): «El contexto geomorfológico en las inundaciones de la ribera del Júcar». Cuadernos de Geografía, nº 67-68, 241-259.

MATHER, P.M. (1979): Theory and quantitative methodes in geomorphology». Progress in Physical Geography, n 3( 4), 471-487.

MICHELETTI, N., CHANDLER, J.H. y LANE, S.N. (2014): «Investigating the geomorphological potential of freely available and accessible Structure-from-Motion photogrammetry using a smartphone». Earth Surface Processes and Landforms, n 40 (4), 473-486.

MORISAWA, M. (1968): Streams. Their dynamics and morphology. New York. McGraw-Hill.

MORISAWA, M. (1973): Fluvial geomorphology. London. George Allen and Unwin.

MORISAWA, M. (1976): Geomorphology laboratory manual. With report forms. New York. John Wiley.

MORISAWA, M. (1985): Rivers. Form and process. K. M. Clayton, University of East Anglia, Geomorphology Texts.

MORISAWA, M. y CLAYTON, K.M. (1985): Rivers. Form and process. London. Longman.

MURRAY, A.B., LAZARUS, E., ASHTON, A., BAAS, A., COCO, G., COULTHARD, T., FONSTAD, M., HAFF, P., MCNAMARA, D., PAOLA, C., PELLETIER, J. y REINHARDT, L. (2009): «Geomorphology, complexity, and the emerging science of the Earth's surface». Geomorphology, nº 103, 496-505.

NAIMAN, R.J. y BILBY, R.E. (1998): River ecology and management. Lessons from the Pacific coastal ecoregion. New York. Springer-Verlag.

NEWSON, M.D. (1992): Land, water and development. River basin systems and their sustainable management. London. Routledge.

NEWSON, M.D. y LARGE, A.R.G. (2006): «'Natural' rivers, 'hydromorphological quality' and river restoration. A challenging new agenda for applied fluvial geomorphology». Earth Surface Processes and Landforms, $\mathrm{n}^{\circ}$ 31, 1.606-1.624.

NÖE, G. (DE LA) y MARGERIE, E. (DE) (1888): Les formes du terrain. Paris. Imprimerie Nationale.

OGLESBY, R.T., CARLSON, C.A. y MCCANN, J.A. (1972): River ecology and man. Proceedings. New York. Academic Press.

OLDROYD, D.R. y GRAPES, R.H. (2008): «Contributions to the history of geomorphology and Quaternary geology: an introduction» in History of Geomorphology and Quaternary Geology (Oldroyd, D.R., Grapes, R.H. y Grigelis, A., Eds.). London, Geological Society, Special Publications, $\mathrm{n}^{\mathrm{o}}$ 301, 1-17.

OLLERO A. (2010): «Channel changes and floodplain management in the meandering middle Ebro River, Spain». Geomorphology, nº 117, 247-260.

OLLERO, A., IBISATE, A., ACÍN, V., BALLARÍN, D., BESNE, P., DÍAZ, E., FERRERBOIX, C., GRANADO, D., HERRERO, X., HORACIO, J., MARTÍN VIDE, J.P., MESANZA, A., MORA, D. y SÁNCHEZ, I. (2014): «Geomorfología y restauración fluvial: seguimiento del derribo de presas en Gipuzkoa». Cuadernos de Investigación Geográfica, no 40 (1), 67-88. 
OLlERO, A., IBISATE, A., GONZALO, L.E., ACÍN, V., BALLARÍN, D., DÍAZ, E., DOMENECH, S., GIMENO, M., GRANADO, D., HORACIO, J., MORA, D. y SÁNCHEZ FABRE, M. (2011): «The IHG index for hydromorphological quality assessment of rivers and streams: updated version». Limnetica, $\mathrm{n}^{\circ} 30$ (2), 255-262.

ORTEGA, J. A. y GARZÓN, G. (2009): «Geomorphological and sedimentological analysis of flash-flood deposits, the case of the 1997 Rivillas flood (Spain) ». Geomorphology, n 112, 1-14.

ORTEGA, J.A. y DURÁN, J.J. (2010): Patrimonio geológico: los ríos en roca de la Península Ibérica. Madrid. Instituto Geológico y Minero de España.

ORTEGA, J.A., RAZOLA, L. y GARZÓN, G. (2014): «Recent human impacts and change in dynamics and morphology of ephemeral rivers». Natural Hazards and Earth System Sciences, $\mathrm{n}^{\circ} 14,713-730$.

PARDÉ, M. (1934): «Fleuves et rivières». Les Études rhodaniennes, no 10(1-2), 97-100.

PASSARGE, S. (1912): Physiologische Morphologie. Hamburgo. Friedeticksen.

PEDRAZA, J. (1996): Geomorfología. Principios, métodos y aplicaciones. Madrid. Editorial Rueda.

PELTIER, L.C. (1950): «The geographic cycle in periglacial regions as it is related to climat geomorphology». Ann. Ass. Amer. Geog., n 40, 219-236.

PENCK, A. (1894): Morphologie der Erdoberflache. Stuttgart, J. Engelhorn.

PENCK, A. (1905): «Climatic features in the land surface». Amer. J. Sci., nº XIX, pp. 165174.

PETTS, G.E. (1984): Impounded rivers. Perspectives for ecological management. Chichester. John Wiley \& Sons Ltd.

PETTS, G.E. y FOSTER, I.D. (1985): Rivers and Landscape. London. Arnold.

PITTY, A.F. (1982): The nature of Geomorphology. London. Methuen.

PLAYFAIR, J. (1802): Illustrations of the Huttonian Theory of the Earth. Edimburgo, William Creech edit.

POWELL, J.W. (1875): Exploration of the Colorado River of the West. New York. Dover.

RHOADS, B.L. (1994): «Short communication on being a 'Real' Geomorphologist». Earth Surface Processes and Landforms, no 19, 269-272.

RHOADS, B.L. y THORN, C.E. (1993): Geomorphology as science. The role of theory. Geomorphology, $\mathrm{n}^{\circ}$ 6, 287-307.

RHOADS, B.L. y THORN, C.E. (1994): «Contemporary philosophical perspectives on physical geography with emphasis on geomorphology». Geographical Review, no 84(1), 90-101.

RHOADS, B.L. y THORN, C.E. (1996): The Scientific Nature of Geomorphology. Chichester. Wiley.

RICHARDS, K. (1982): Rivers. Form and process in alluvial channels. London. Methuen.

RICHTHOFEN, F. VON (1886): Fiihrer fur Forschungsreisende. Anleitung zu Beobachtungen iiber Gegenstande der physischen Geographie und Geologie. Hannover. G. Janecke.

RINALDI, M., GRANT, G., KONDOLF, M. y PIÉGAY, H. (2008): «Restoration and management of physical processes and sediments». 4th ECRR International Conference on River restoration, Chapter 6, Session 5, 377-550.

RITTER, D.F. (1978): Process geomorphology. Dubuque. W. C. Brown. 
RITTER, D.F., KOCHEL, R.C. y MILLER, J.R. (1995): Process geomorphology. Wm. C. Brown.

ROMERO-DÍAZ, A. y LÓPEZ-BERMÚDEZ, F. (1987): «Morfometría de redes fluviales. Revisión crítica de los parámetros más utilizados y aplicación al alto Guadalquivir». Papeles de Geografía (Física), no 12, 47-62.

ROSGEN, D.L. (1996): Applied river morphology. Iowa. Wildland Hydrology Books.

RUBEY, A. (1938): The force required to move particles on a stream bed. U.S. Geol. Surv. Prof. Paper, 189-E.

SACK, D. (2002): «The educational of the history of geomorphology. Geomorphology, ${ }^{\circ}$ 47, 313-323.

SALA, M. (1982): «Datos cuantitativos de los procesos geomórficos fluviales actuales en la cuenca de la riera de Fuirosos (Montnegre, Macizo Litoral Catalán)». Cuadernos de Investigación Geográfica, n ${ }^{\circ}$ VIII, 1-2, 51-68.

SALA, M. (1984): «Geomorfología actual. Guía conceptual, temática y bibliográfica». Revista de Geografía, $\mathrm{n}^{\circ}$ XVIII, 209-244.

SALA, M. y BATALLA, R.J. (1996): Teoría y métodos en Geografía Física. Madrid. Ed. Síntesis.

SÁNCHEZ MARTÍNEZ, F.J. y LASTRA, J. (2011): Guía metodológica para el desarrollo del Sistema Nacional de Cartografía de Zonas Inundables. Madrid. Ministerio de Medio Ambiente y Medio Rural y Marino.

SANJUÁN, Y., GARCÍA RUIZ, J.M., GÓMEZ VILLAR, A., NADAL-ROMERO, E., ÁLVAREZ MARTÍNEZ, J., SERRANO, P., ARNÁEZ, J. y GONZÁLEZ-SAMPÉRIZ, P. (2014): «Cambios geomorfológicos en cauces torrenciales en relación con cambios» en la cubierta vegetal en Avances de la Geomorfología en España 2012-2014 (Schnabel, S. \& Gómez, A., eds.). Cáceres, XIII Reunión Nacional de Geomorfología, 79-82.

SCHEIDEGGER, A.E. (1961): Theoretical Geomorphology. Berlin. Springer.

SCHEIDEGGER, A.E. (1982): Principles of geodynamics. Berlin. Springer-Verlag..

SCHEIDEGGER, A.E. (1987): Systematic geomorphology. New York. Springer-Verlag Wien.

SCHMITT, L. (2001): Typologie hydro-geomorphologique fonctionnelle de cours d'eau. Recherche methodologique appliquee aux systemes fluviaux d'Alsace. Tesis doctoral, Université Louis Pasteur, Strasbourg I.

SCHUMM, S.A. (1956): «Evolution of drainage systems and slopes in badlands ut Perth Amboy, New Jersey». Bull. Geol. Soc. Arn., n 67, 597-646.

SCHUMM, S.A. (1972): River morphology. Dowden. Benchmark Papers in Geology., Hutchinson \& Ross, Inc.

SCHUMM, S.A. (1977): The Fluvial System. New York. Wiley.

SCHUMM, S.A. (1979): «Geomorphic thresholds. The concept and its applications». Transactions of Institute of British Geographers, $\mathrm{n}^{\circ}$ 4, 485-515.

SCHUMM, S.A. (2005): River variability and complexity. Cambridge. Cambridge University Press.

SCHUMM, S.A. y LICHTY, R.W. (1965): «Time, space and causality in geomorphology». American Journal of Science, $\mathrm{n}^{\mathrm{o}} 263,110-119$. 
SEAR, D.A., NEWSON, M.D. y THORNE, C.R. (2003): Guidebook of applied fluvial geomorphology. London. RYD Technical Report, DEFRA y Environment Agency.

SEGURA, F. y SANCHIS, C. (2013): «Assessment of channel changes in a Mediterranean ephemeral stream since the early twentieth century. The Rambla de Cervera, eastern Spain». Geomorphology, n² 201, 199-214.

SHERMAN, L.K. (1932): «Flow from rainfall by the unit-graph method». Engineering News-Record, $\mathrm{n}^{\mathrm{o}}$ 108, 501-505.

SHIELDS, A. (1936): «Application of similarity principles and turbulence research to bedload movement». Mitteilunger der Preussischen Versuchsanstalt für Wasserbau und Schiffbau, $\mathrm{n}^{\circ} 26,5-24$.

SIMON, A., BENNETT, S.J. y CASTRO J.M. (2013): Stream Restoration in Dynamic Fluvial Systems. Scientific Approaches, Analysis, and Tools. American Geophysical Union, Geophysical Monograph Series.

SNCZI (2011): Guía Metodológica para el desarrollo del Sistema Nacional de Cartografía de Zonas Inundables. Madrid. Ministerio de Medio Ambiente y Medio Rural y Marino.

SPARKS, B.W. (1972): Geomorphology. London. Longman.

STODDART, D.R. (1997): Process and form in Geomorphology. London. Routledge.

STOKES M., GRIFFITHS, J.S. y MATHER, A. (2012): «Palaeoflood estimates of Pleistocene coarse grained river terrace landforms (Rio Almanzora, SE Spain) ». Geomorpho$\log y, \mathrm{n}^{\circ} 149,11-26$.

STOTT, T. (2010): «Fluvial geomorphology». Progress in Physical Geography, no 34(2), 221-245.

STRAHLER, A.N. (1950): «Davi's concept of slope development viewed in the light of recent quantitative investigations». American Association of Geographers Annals, $\mathrm{n}^{\circ} 40$, 209-213.

STRAHLER, A.N. (1951): Physical geography. Nueva York. Wiley.

STRAHLER, A.N. (1952): «Dynamic basis of geomorphology». Geological Society of America Bulletin, $\mathrm{n}^{\circ}$ 66, 923-938.

STRAHLER, A.N. (1954): «Statistical analysis in geomorphic». Research. Jour. Geol., n ${ }^{\circ}$ LXII, 1-25.

STRAHLER, A.N. (1956a): «Dimensional analysis in geomorphology». Bull. Geol. Soc. Arn., $\mathrm{n}^{\circ}$ LXVII, 521-599.

STRAHLER, A.N. (1956b): «Quantitative slope analysis». Geological Society of America Bulletin, $\mathrm{n}^{\circ}$ 67, 571-596.

STRAHLER, A.N. (1964): «Quantitative geomorphology of drainage basins and channels networks» in Handbook of applied hydrology (Chow, V.T., Ed.). New York, McGraw Hill.

STRAHLER, A.N. (1968): «Quantitative Geomorphology» in The Encyclopedia of Geomorphology (Fairbridge, R.W., Ed.). Stroudsburg, Dowden, Hutchinson and Ross.

STRAHLER, A.N. (1980): «Systems theory in Physical Geography». Physical Geography, $\mathrm{n}^{\mathrm{o}} 1,1-27$.

STRAHLER, A.N. (1992): Geología Física. Barcelona. Ediciones Omega.

SUMMERFIELD, M.A. (1991): Global geomorphology. An introduction to the study of landforms. Essex. Longman, Harlow. 
TAROLLI, P. (2014): «High-resolution topography for understanding Earth surface processes. Opportunities and challenges». Geomorphology, n 216, 295-312.

THORN, C.E. (1982): Space and time in geomorphology. Proceedings of the 12th Binghamton symposium. London. George Allen and Unwin.

THORNBURY, W.D. (1954): Principles of Geomorphology. New York. Wiley.

THORNDYCRAFT, V. R., BENITO, G. y GREGORY, K.J. (2008): «Fluvial geomorphology. A perspective on current status and methods». Geomorphology, n 98, 2-12.

THORNE, C.R., BATHURST, J.C. y HEY, R.D. (1987): Sediment transport in gravel-bed rivers. New York. Wiley.

THORNE, C.R., HEY, R.D. y NEWSON, M.D. (1997): Applied Fluvial Geomorphology for River Engineering and Management. Chichester, Wiley.

THORNES, J.B. (1978): «The character and problems of theory in contemporary Geomorphology» in Geomorphology. Present problems and future prospects (Embleton, C., Brunsden, D. y Jones, D.K.C., Eds.). Oxford, British Geomorphological Research Group, Oxford University Press, 14-24.

THORNES, J.B. (1979): River channels. London. Nelson Thornes Ltd.

THORNES, J.B. y BRUNSDEN, D. (1977): Geornorphology and time. London. Methuen.

TINKLER, K.J. (1985): A short history of Geomorphology. Rowman \& Littlefield Publishers.

TINKLER, K.J. y WOHL, E.E. (1998): Rivers over rock. fluvial processes in bedrock channels. Florida. Geophysical monograph, American Geophysical Union, Editor American Geophysical Union.

TRICART, J. (1952): «La géomorphologie et le notion d'echelle». Revue de Géomorphologie Dynamique, $\mathrm{n}^{\circ}$ 3, 213-218.

TRICART, J. (1953): «La géomorphologie et les homes». Revue de Géomorphologie Dynamique, $\mathrm{n}^{\circ} 4,153-156$.

TRICART, J. (1962): L'épiderme de la Terre. Esquisse d'une geornorphologie appliquée. Paris. Masson.

TRICART, J. (1965): Principes et Méthodes de la Geomorphologie. Paris. Masson.

URIBELARREA, D. (2008): Dinámica y evolución de las llanuras aluviales de los ríos Manzanares, Jarama y Tajo, entre las ciudades de Madrid y Toledo. Tesis doctoral, Universidad Complutense de Madrid, Madrid.

VERICAT, D. y BATALLA, R.J. (2010): «Sediment transport from continuous monitoring in a perennial mediterranean stream». Catena, $\mathrm{n}^{\circ} 82,77-86$.

VERSTAPPEN, H.T. (1983): Applied geomorphology. geomorphological surveys for environmental development. New York. Elsevier.

VITEK, J.D. (2013): Geomorphology. «Perspectives on Observation, History, and the Field Tradition». Geomorphology, n 200, 20-33.

VOGEL, R. (2011): «Hydromorphology». Journal of Water Resources Planning and Management, $\mathrm{n}^{\mathrm{o}} 137$ (2), 147-149.

WEST, E.A. (1978): The equilibrium of natural streams. Norwich. Geo Abstracts.

WESTOBY, M.J., BRASINGTON, J., GLASSER, N.F., HAMBREY, M.J. y REYNOLDS, J.M. (2012): «Structure-from-motion' photogrammetry: a low-cost, effective tool for geoscience applications». Geomorphology, n 179, 300-314. 
WHEATON, J.M. (2008): Uncertainty in morphological sediment budgeting of rivers. Tesis doctoral, University of Southampton, Southampton.

WHITTON, B.A. (1975): River ecology. Berkeley. University of California Press.

WOHL, E.E. (2000): Mountain rivers. Water resources monograph, Editor American Geophysical Union.

WOHL, E.E. (2013): «The complexity of the real world in the context of the field tradition in geomorphology». Geomorphology, nº 200, 50-58.

WOLMAN, M.G. y MILLER, J.P. (1960): «Magnitude and frequency of forces in geomorphic processes». Journal of Geology, $\mathrm{n}^{\circ}$ 68, 54-74.

WOODGET, A.S., CARBONNEAU, P.E., VISSER, F. y MADDOCK, I.P. (2014): «Quantifying submerged fluvial topography using hyperspatial resolution UAS imagery and structure from motion photogrammetry». Earth Surface Processes and Landforms, ${ }^{\circ} 40$ (1), 47-64.

WYZGA, B. (1993): «River response to channel regulation. Case study of the Raba river, Carpathians, Poland». Earth Surface Processes and Landforms, $n^{\circ} 18$ (6), 541-556.

WYZGA, B. (1996): «Changes in the magnitude and transformation of flood waves subsequent to the channelization of the Raba River, Polish Carpathians». Earth Surface Processes and Landforms, $\mathrm{n}^{\mathrm{o}} 21$ (8), 749-763.

ZĂVOIANU, I. (1985): Morphometry of drainage basins. Bucharest. Developments in Water Science, Elsevier. 